\title{
ПОСТРОЕНИЕ ТУННЕЛЬНЫХ ОСЕСИММЕТРИЧНЫХ СВЕРХЗВУКОВЫХ ВОЗДУХОЗАБОРНИКОВ
}

\author{
Галкин Владислав Михайлович, \\ канд. физ.-мат. наук, ст. науч. сотр, доцент каф. геологии \\ и разработки нефтяных месторождений Института природных ресурсов \\ Томского политехнического университета, Россия, 634050, г. Томск, \\ пр. Ленина, 30. Email: vlg@tpu.ru \\ Звегинцев Валерий Иванович, \\ д-р техн. наук, гл. науч. сотр. Института теоретической и прикладной \\ механики им. С.А. Христиановича СО РАН, Россия, 630090, г. Новосибирск, \\ ул. Институтская, 4/1. Email: zvegin@itam.nsc.ru
}

\begin{abstract}
Актуальность работы. Эффективность преобразования химической энергии топлива в механическое движение сверхзвукового летательного аппарата определяется потерями механической энергии на сопротивление летательного аппарата и потерями полного давления при торможении потока в воздухозаборнике воздушно-реактивного двигателя. Поэтому при прочих равных условиях более энергоэффективным будет тот летательный аппарат, у которого эти потери меньше. Существенное повышение энергоэффективности ожидается от применения осесимметричных изоэнтропических туннельных воздухозаборников. В рамках модели невязкого течения предлагается численная методика построения указанных воздухозаборников с профилированным центральным телом и цилиндрической обечайкой.

Цель работы. В настоящее время отсутствуют способы профилирования осесимметричных изоэнтропических туннельных воздухозаборников, не имеющих элементов, выступающих за цилиндрический корпус летательного аппарата. Поэтому целью исследования является разработка методики построения таких воздухозаборников.

Методы исследования. Используется численная реализация метода характеристик при условии изоэнтропичности течения. Предлагаемая методика состоит из двух задач. В первой задаче от угловой точки строится контур центрального тела с заданной ординатой точки фокусировки характеристик. Во второй задаче производится построение оставшейся части контура до другой угловой точки, при этом используется обращенное течение в кольцевом сопле с цилиндрической обечайкой. Одновременно с решением второй задачи определяется положение обечайки. Центральное тело может содержать на краях по угловой точке. При использовании промежуточной линии тока центральное тело будет гладким.

Результаты. Создана методика для расчета семейства контуров осесимметричных туннельных сверхзвуковых воздухозаборников, геометрические характеристики которых однозначно описываются исходными данными.
\end{abstract}

\section{Ключевые слова:}

Энергоэффективность, сверхзвуковое течение, туннельный осесимметричный воздухозаборник, идеальный совершенный газ, метод характеристик, изоэнтропическое течение.

\section{Введение}

Требование энергоэффективности и инженерные ограничения в большинстве случаев приводят к тому, что при проектировании сверхзвуковых летательных аппаратов с воздушно-реактивным двигателем желательно иметь корпус цилиндрической формы с минимальным количеством выступающих элементов [1]. Этому требованию удовлетворяет воздухозаборник, расположенный в носовой части, и туннельный воздухозаборник (ТуВ). Иногда ТуВ называется утопленным воздухозаборником. Пик исследований воздухозаборников, расположенных в носовой части, пришелся на 1950-1960-е гг. [1-5], что связано с переходом авиации на воздушно-реактивные двигатели. В настоящее время осесимметричный воздухозаборник широко применяется в летательных аппаратах. В современной литературе [6-11] его проектирование подробно освещено, поэтому в данной статье этот вопрос не рассматривается.

Что касается ТуВ, по-видимому, первое его упоминание относится к дозвуковому турбореактивному самолету Messerschmitt P.1110 [12, 13], спроектированному в Германии в начале 1945 г. Одна- ко в производство этот самолет не пошел. Интерес к ТуВ в последующие годы был невысок, прежде всего, из-за больших потерь полного давления, что связано с использованием простейших методик для его построения.

В последнее время интерес к ТуВ начал возвращаться, о чем свидетельствуют публикации. В работе [14] предложена конструкция воздухозаборника для дозвукового аппарата, которая не пригодна при сверхзвуковом течении. Рассмотренная в [15] компоновка предназначена для сверхзвуковых скоростей, однако используемый одномерный подход позволяет получить лишь простейшие оценки утопленного воздухозаборника и не может учесть эффекты, связанные с осесимметричностью течения. Тем более что предлагаемый в этой статье подход не позволяет получить однозначный облик ТуВ.

Методика построения ТуВ, рассматриваемая в данной статье, основана на уравнениях, описывающих течение осесимметричного сверхзвукового идеального (невязкого нетеплопроводного) совершенного газа с использованием метода характеристик. При этом для каждого набора исходных 

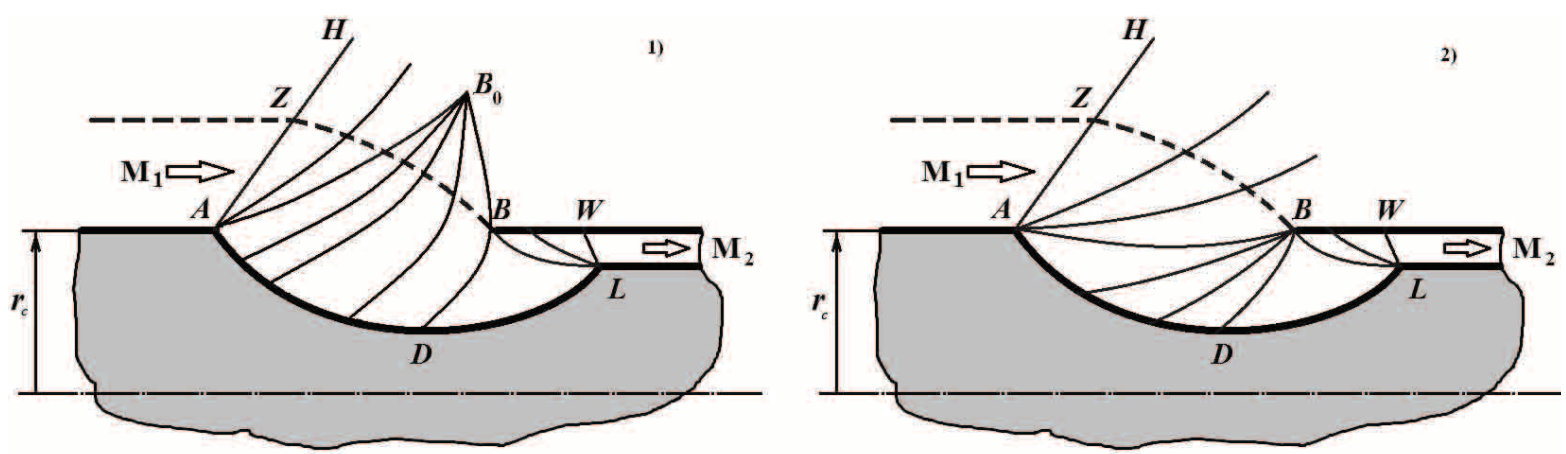

Pис. 1. Схема сверхзвукового осесимметричного туннельного воздухозаборника: (1) точка фокусировки характеристик $B_{0}$ находится выше обечайки; (2) точка фокусировки характеристик В находится на обечайке. $M_{1}$ - число Маха внешнего равномерного потока; $M_{2}$ - число Маха равномерного потока в минимальном сечении ТуВ; $A H$ - начальная равномерная характеристика; A - угловая точка на входе в Тув; $r_{c}$ - радиус цилиндрического корпуса и цилиндрической обечайки; $B W$ - элемент обечайки; $A D L$ - искомый профиль центрального тела; L - угловая точка на центральном теле в минимальном сечении; LW - равномерная характеристика в минимальном сечении; BL - характеристика, замыкающая веер характеристик; BD - характеристика, ограничивающая область течения под обечайкой; ZB - линия тока (граница захватываемой струи)

Fig. 1. Diagram of supersonic ducted axisymmetric air inlet: (1) focusing point of $B_{0}$ characteristics is upper than the shell; (2) focusing point of $B$ characteristics is on the shell. $M_{1}$ is the Mach number of the external smooth flow; $M_{2}$ is the Mach number of the smooth flow in minimal cut set of ducted air inlet; $A H$ is the initial uniform characteristic; $A$ is the angular point at the input of the ducted air inlet; $r_{c}$ is the radius of cylindrical body and cylindrical shell; BW is the shell element; $A D L$ is the desired profile of the central body; $L$ is the angular point on the central body in minimal cut set; $L W$ is the uniform characteristic in minimal cut set; $B L$ is the characteristic closing the fan of characteristics; $B D$ is the characteristic limiting the flow range under the shell; $Z B$ is the current line (boundary of captured jet)

данных строится единственный профиль воздухозаборника. Окончательный вывод о пригодности формы ТуВ получается после расчета течения в нем вязкого газа аналогично работе [16].

ТуВ предлагаемой схемы имеет цилиндрический корпус и такого же радиуса обечайку. Профиль центрального тела гладкий, за исключением, быть может, начальной и конечной угловых точек, которые можно сгладить путем использования промежуточных линий тока в качестве профиля центрального тела. Рис. 1 демонстрирует два варианта ТуВ с разным положением точек фокусировки.

Схема течения в ТуВ имеет следующий вид. Через отверстие $A B$ внешний равномерный поток, имеющий число Маха $\mathrm{M}_{1}$, начинает втекать, одновременно разворачиваясь и ускоряясь около угловой точки $A$. Далее при обтекании центрального тела на участке $A D$ происходит замедление и поворот потока таким образом, что характеристики, выходящие с центрального тела, пересекаются в заданной точке. Это будет точка $B_{0}$ на левом рис. 1 и соответственно точка $B$ на правом рис. 1 . При обтекании участка $D L$ газ замедляется и выравнивается так, что на характеристику $L W$ приходит равномерный параллельный поток с числом Maха $\mathrm{M}_{2}$. При этом полагается, что течение в ТуВ происходит изоэнтропически при числах Маха больше 1.

Построение профиля центрального тела $A D L$ разделяется на две задачи. В первой задаче строится участок $A D$ путем интерполяции параметров вдоль характеристик на найденное значение расхода. Во второй задаче для построения участка $D L$ используется обращенное (повернутое на $180^{\circ}$ ) течение в кольцевом осесимметричном сопле, схема которого показана на рис. 2. Эта задача сводится к построению сверхзвукового сопла, состоящего из цилиндрической обечайки и профилированного центрального тела при заданной характеристике $B D$ на выходе и равномерной характеристике $L W$ на входе. Прием обращения течения возможен, поскольку выполняется условие изоэнтропичности течения и тип уравнений, описывающих течение, остается гиперболическим.

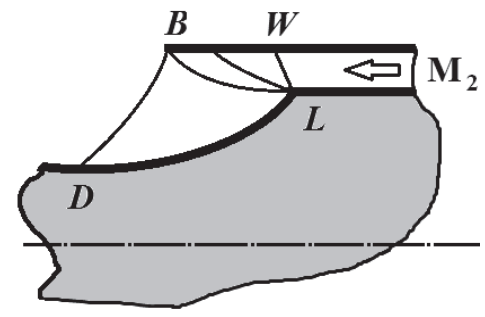

Рис. 2. Элемент туннельного воздухозаборника. Схема участка кольцевого осесимметричного сопла

Fig. 2. Element of the ducted air inlet. Diagram of a section of the ring axisymmetric nozzle

\section{Математическая постановка задачи}

Рассматривается осесимметричное сверхзвуковое течение идеального (невязкого нетеплопроводного) совершенного газа. Контур центрального тела находится методом характеристик. Для этого аналогично [17] используются уравнения характеристик $C^{ \pm}$:

$$
\frac{d r}{d x}=\operatorname{tg}(\theta \pm \alpha)
$$

условия совместности на характеристиках: 


$$
d \theta \pm \frac{\cos ^{2} \alpha}{(k+1) / 2-\cos ^{2} \alpha} d \alpha \pm \frac{\sin \alpha \sin \theta}{r \cos (\theta \pm \alpha)} d x=0
$$

где $x$ и $r$ - продольная и поперечная координаты; $\alpha$ - угол Маха; $\theta$ - угол наклона вектора скорости к оси $x ; k$ - показатель адиабаты. Расход через элемент характеристики находится из выражения:

$$
G=2 \int_{r_{1}}^{r_{2}} \rho w r \frac{\sin \alpha}{\sin (\theta \pm \alpha)} d r
$$

где $G$ - расход через элемент характеристики, лежащий между точками 1 и $2 ; w^{2}=u^{2}+v^{2}-$ модуль скорости; $u$ и $v$ - проекции вектора скорости на оси $x$ и $r ; \rho$ - плотность; $M=\frac{1}{\sin \alpha}-$ число Маха. Если $\alpha=\arcsin (1 / M)=$ const и $\theta=0$, то соотношение (3) приводится к виду:

$$
G=M\left(\frac{k+1}{M^{2}(k-1)+2}\right)^{\frac{k+1}{2(k-1)}}\left(r_{2}^{2}-r_{1}^{2}\right),
$$

В указанных формулах используются безразмерные величины $w, u, v, x, r, \rho, G$. Они получены следующим образом:

$$
\begin{gathered}
w=\frac{w_{\text {dim }}}{a_{*}}, u=\frac{u_{\text {dim }}}{a_{*}}, v=\frac{v_{\text {dim }}}{a_{*}}, x=\frac{x_{\text {dim }}}{r_{c}}, \\
r=\frac{r_{\text {dim }}}{r_{c}}, \rho=\frac{\rho_{\text {dim }}}{\rho_{*}}, G=\frac{G_{\text {dim }}}{\pi \rho_{*} a_{*} r_{c}^{2}},
\end{gathered}
$$

где индекс $\operatorname{dim}$ соответствует размерным величинам; $r_{c}$ - размерный радиус цилиндрического корпуса; $a_{*}-$ размерная критическая скорость; $\rho_{*}-$ размерная критическая плотность. Абсцисса точки $A$ на рис. 1 полагается равной 0.

Неизвестные величины находятся из системы 4-х нелинейных уравнений, которые получены из конечно-разностной записи уравнений $(1),(2)$ :

$$
\left\{\begin{aligned}
\frac{r_{3}^{j}-r_{2}}{x_{3}^{j}-x_{2}} & =\operatorname{tg}\left(\theta_{23}-\alpha_{23}\right), \\
\frac{r_{3}^{j}-r_{1}}{x_{3}^{j}-x_{1}} & =\operatorname{tg}\left(\theta_{13}+\alpha_{13}\right), \\
\theta_{3}^{j}-\theta_{1} & +\frac{\cos ^{2} \alpha_{13}}{(k+1) / 2-\cos ^{2} \alpha_{13}}\left(\alpha_{3}^{j}-\alpha_{1}\right)+ \\
& +\frac{\sin \alpha_{13} \sin \theta_{13}}{r_{13} \cos \left(\theta_{13}+\alpha_{13}\right)}\left(x_{3}^{j}-x_{1}\right)=0, \\
\theta_{3}^{j}-\theta_{2} & -\frac{\cos ^{2} \alpha_{23}}{(k+1) / 2-\cos ^{2} \alpha_{23}}\left(\alpha_{3}^{j}-\alpha_{2}\right)- \\
& +\frac{\sin \alpha_{23} \sin \theta_{23}}{r_{23} \cos \left(\theta_{23}-\alpha_{23}\right)}\left(x_{3}^{j}-x_{2}\right)=0,
\end{aligned}\right.
$$

здесь индексы 1,2 соответствуют известным параметрам на характеристиках $C^{ \pm}$; индекс 3 соответствует искомым параметрам в точке пересечения характеристик; $j=1,2 . . .-$ номер итерации. Обозначим $p=\{\alpha, \theta, x, r\}$, тогда $p_{13}=\left(p_{1}+p_{3}{ }^{j-1}\right) / 2, p_{23}=\left(p_{2}+p_{3}{ }^{j-1}\right) / 2$. По- лученная система уравнений решалась итерационно. Итерации оканчивались при выполнении условия $\max \left|p_{3}{ }^{j}-p_{3}{ }^{j-1}\right|<10^{-6}$ для $j>1$. На начальной итерации полагалось $p_{3}{ }^{0}=\left(p_{1}+p_{2}\right) / 2$. Более подробно схема решения описана в [18] и [19].

Уравнение (3) для вычисления расхода интегрируется численно по формуле средних прямоугольников.

В угловых точках, а также в точках фокусировки уравнение (2) интегрируется в квадратурах и превращается в формулу Прандтля-Майера, которая выполняется как при ускорении, так и при замедлении потока:

$$
\begin{gathered}
\theta_{1} \pm \omega\left(\alpha_{1}\right)=\theta_{2} \pm \omega\left(\alpha_{2}\right), \\
\omega(\alpha)=-\alpha+\sqrt{\frac{k+1}{k-1}} \operatorname{arctg}\left(\sqrt{\frac{k+1}{k-1}} \operatorname{tg} \alpha\right),
\end{gathered}
$$

где индексы 1 и 2 соответствуют параметрам до и после разворота.

\section{Алгоритм построения профиля центрального тел}

Пусть заданы следующие независимые параметры: $N$ - число узлов сетки на характеристиках; $k$ - показатель адиабаты; $M_{1}$ - число Маха во внешнем равномерном потоке; $M_{2}$ - число Маха равномерного потока в минимальном сечении; $r_{h}-$ opдината точки $H$; $r_{B_{0}}$ - ордината точки $B_{0}$. Для $r_{B_{0}}=1$ и $r_{B_{0}}>1$ алгоритмы различаются.

Случай $r_{B_{0}}>\mathbf{1}$. Схема построения методом характеристик профиля центрального тела, изображенного на левом рис. 1 , имеет следующий вид:

1. По формуле (1) строится равномерная характеристика $A H$ с параметрами в узлах сетки $\alpha=\arcsin \left(1 / M_{1}\right), \theta=0$.

2. От характеристики $A H$ по формулам (5) рассчитываются характеристики в центрированной волне разрежения около точки $A$, пока очередная характеристика $A B_{0}$ не будет иметь значение ординаты в своей крайней точке $B_{0}$, равной $r_{B_{0}}$.

3. От характеристики $A B_{0}$ по формулам (5) рассчитываются характеристики в центрированной волне около точки $B_{0}$, пока на очередной характеристике $B_{0} D$ не появится точка со значениями $\theta=0$ и $r=1$. Это будет точка $B$ начала обечайки.

4. По формуле (3) вычисляется расход $G_{B_{0} D}$ через характеристику $B_{0} D$.

5. По формуле (3) вычисляется расход $G_{B_{0} B}$ через характеристику $B_{0} B$.

6. Вычисляется расход воздухозаборника $\mathrm{G}=G_{B_{0} D}-G_{B_{0} B}$.

7. Повторяются пункты 2 и 3 . При этом вдоль найденных характеристик вычисляются параметры на искомом контуре. Для этого используется формула (3) и квадратичная интерполяция на расход $G$.

8. Запоминается $\alpha_{b}$ - значение угла Маха на характеристике $B_{0} D$ в точке $B$.

9. Из формулы (4) рассчитывается ордината минимального сечения: 


$$
r_{L}=\sqrt{1-\frac{G}{M_{2}}\left(\frac{k+1}{M_{2}^{2}(k-1)+2}\right)^{\frac{k+1}{2(1-k)}}} .
$$

10. По формуле (1) строится равномерная характеристика $L W$ с параметрами в узлах сетки $\alpha=\arcsin \left(1 / M_{2}\right), \theta=0$.

11.0т характеристики $L W$, как показано на рис. 2 , по формулам (5) рассчитываются характеристики в центрированной волне разрежения около точки $L$, пока угол Маха на очередной характеристике $L B$ в точке пересечения с обечайкой не станет равным $\alpha_{b}$. При этом получается длина обечайки от минимального сечения до точки $B$.

12.В характеристическом треугольнике $L B D$ по характеристикам $B D$ и $L B$ решается задача Гурса. Для этого используются формулы (5).

13. Параметры на искомом контуре $L D$ находятся квадратичной интерполяцией на расход $G$ вдоль характеристик при решении задачи Гурса. Для этого используется формула (3).

Случай $\boldsymbol{r}_{B_{0}}=\mathbf{1}$. Схема построения методом характеристик профиля центрального тела, изображенного на правом рис. 1, имеет следующий вид:

1. По формуле (1) строится равномерная характеристика $A H$ с параметрами в узлах сетки $\alpha=\arcsin \left(1 / M_{1}\right), \theta=0$.

2. От характеристики $A H$ по формулам (5) рассчитываются характеристики в центрированной волне разрежения около точки $A$, пока очередная характеристика $A B$ не будет иметь значение ординаты в своей крайней точке $B$, равной 1 .

3. От характеристики $A B$ по формулам (5) рассчитываются характеристики в центрированной волне около точки $B$, пока на очередной характеристике $B D$ в точке $B$ не выполнится условие $\theta=0$. Это будет начало обечайки.

1)

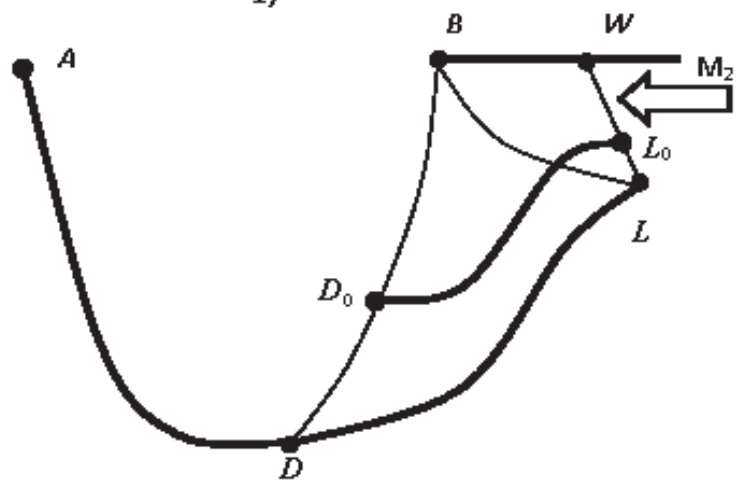

4. По формуле (3) вычисляется расход $G$ через характеристику $B D$. Далее пункты аналогичны пп. 7-13 предыдущего алгоритма, за исключением того, что точки $B_{0}$ и $B$ совпадают.

\section{Построение профиля по промежуточной линии тока}

Если требуется построить центральное тело без угловых точек, то используются промежуточные линии тока. При этом для однозначного определения нужной линии тока задается относительный расход $G_{R E L}$, который находится из соотношения:

$$
G_{R E L}=\frac{G_{L_{0}}}{G_{L}},
$$

где $G_{L_{0}}$ - расход между обечайкой и искомой линией тока; $G_{L}-$ расход между обечайкой и линией тока с угловой точкой. Значению $G_{R E L}=1$ соответствует контур с угловой точкой, значению $G_{R E L}<1$ соответствует гладкий контур. Очевидно, что в последнем случае длина ТуВ будет больше.

Рассмотрим случай, когда необходимо избавиться от угловой точки $L$ и построить участок $L D$ на центральном теле по промежуточной линии тока. На рис. 3 изображена используемая часть расчетной области с обращенным течением.

Хорошо известен прием, приведенный в [17], для построения сопла с равномерными характеристиками на входе и на выходе. Тогда контур без угловой точки в минимальном сечении строится путем сдвига и растяжения некоторой промежуточной линии тока. В рассматриваемом случае необходимо учитывать, что характеристика $B D$ не является равномерной. Поэтому после решения в треугольнике $D B L$ задачи Гурса линия тока из угловой точки $L$ придет в точку $D$. А линия тока, которая вышла из точки $L_{0}$, придет в точку $D_{0}$, как

Pис. 3. Схема построения участка на центральном теле по промежуточной линии тока: (1) без смещения угловой точки L и с изломом контура; (2) со смещением угловой точки L и гладким контуром. AD - построенный участок; $L D$ - линия тока C угловой точкой; $L_{0} D_{0}$ - гладкая линия тока; $L_{0} D$ - гладкая линия тока (искомый участок центрального тела); BW - элемент обечайки; BD - заданная характеристика; BL - характеристика, замыкающая центрированную волну разрежения; LW - равномерная характеристика

Fig. 3. Diagram of plotting a section on the central body by the intermediate current line: (1) without displacement of the angular point $L$ and with the contour fracture; (2) with displacement of the angular point $L$ and with smooth contour. AD is the plotted section; $L D$ is the current line with the angular point; $L_{0} D_{0}$ is the smooth current line; $L_{0} D$ is the smooth current line (the desired area of the central body); $B W$ is the shell element; $B D$ is the given characteristic; $B L$ is the characteristic closing the centered expansion wave; $L W$ is the uniform characteristic 
показано на левом рис. 3. Так как характеристика $B D$ неравномерная, то параметры в точке $D_{0}$ и в точке $D$ не будут совпадать, в частности $\theta(D) \neq \theta\left(D_{0}\right)$. Следовательно, растяжение и сдвиг линии тока $D_{0} L_{0}$ в точку $D$ приведет к излому контура в этой точке.

Для построения гладкого контура центрального тела предлагается использовать тот факт, что расход $G$ известен, характеристика $L W$ равномерная и в каждой точке на ней $\alpha=$ const и $\theta=$ const. Для заданного расхода $G$ положение ординаты $r_{L_{0}}$ точки $L_{0}$ на характеристике $L W$ фиксировано и определяется по формуле (4):

$$
r_{L_{0}}=\sqrt{1-\frac{G}{M_{2}}\left(\frac{k+1}{M_{2}^{2}(k-1)+2}\right)^{\frac{k+1}{2(1-k)}} .}
$$

Поэтому необходимо сдвинуть угловую точку так, чтобы положение ординаты $r_{L_{0}}$ точки $L_{0}$ соответствовало некоторой гладкой линии тока, как показано на правом рис. 3. Используя формулы (4) и (6), получим:

$$
\begin{gathered}
G_{R E L}=\frac{G_{L_{0}}}{G_{L}}= \\
=\frac{M\left(\frac{k+1}{M^{2}(k-1)+2}\right)^{\frac{k+1}{2(k-1)}}\left(1-r_{L_{0}}^{2}\right)}{M\left(\frac{k+1}{M^{2}(k-1)+2}\right)^{\frac{k+1}{2(k-1)}}\left(1-r_{L}^{2}\right)}=\frac{1-r_{L_{0}}^{2}}{1-r_{L}^{2}} .
\end{gathered}
$$

Следовательно, новое положение ординаты угловой точки $L$ будет:

$$
r_{L}=\sqrt{1-\frac{1-r_{L_{0}}^{2}}{G_{R E L}}} .
$$

Таким образом, при построении профиля по промежуточной линии тока на заданный расход $G_{R E L}$ достаточно пересчитать положение ординаты угловой точки $L$. При этом алгоритмы, описанные выше, не меняются. На расход воздухозаборника значение $G_{R E L}$ не влияет.

Аналогичный прием можно использовать для построения сопла с равномерными характеристиками на входе и на выходе.

\section{Результаты расчетов}

На рис. 4 показаны результаты расчета ТуВ для $N=100, k=1,4 ; \mathrm{M}_{1}=4 ; \mathrm{M}_{2}=1,2 ; r_{h}=1,9 ; r_{B_{0}}=1$; $G_{R E L}=1$. Данному варианту соответствует точка фокусировки $B$, лежащая на обечайке. В качестве интересного факта отметим, что для широкого диапазона исходных данных характеристика $A B$ является параболой.

Для сравнения на рис. 5 показаны результаты расчетов для $N=100, k=1,4 ; M_{1}=4 ; M_{2}=1,2 ; r_{h}=1,9$; $r_{B_{0}}=1,5 ; G_{R E L}=1$. Данному варианту соответствует точка фокусировки $B_{0}$, лежащая выше обечайки.

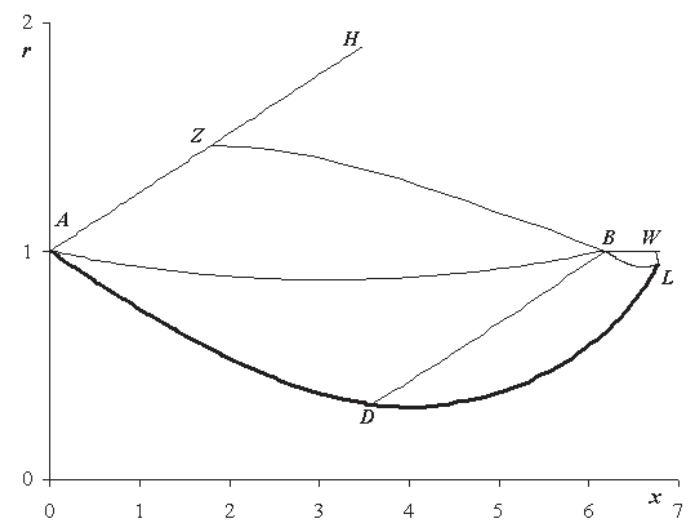

Рис. 4. Результатрасчета контура центрального теладля ТуВ сточкой фокусировки на обечайке. L, A - угловые точки; Bточка фокусировки и начало обечайки; $А Н$ - равномерная характеристика во внешнем потоке; LW - равномерная характеристика в минимальном сечении; ZB - граница захватываемой струи; АВ - характеристика, пересекающая обечайку; DBL - область решения задачи Гурса, $A D L$ - контур центрального тела; BW - элемент обечайки

Fig. 4. Results of designing the central body contour for the ducted air inlet with the focusing point on the shell. L, A are the angular points; $B$ is the focusing point and the beginning of the shell; $A H$ is the uniform characteristic in the external flow; $L W$ is the uniform characteristic in the minimal cut set; $Z B$ is the boundary of the captured jet; $A B$ is the characteristic crossing the shell; $D B L$ is the Goursat problem space; $A D L$ is the central body contour; BW is the shell element

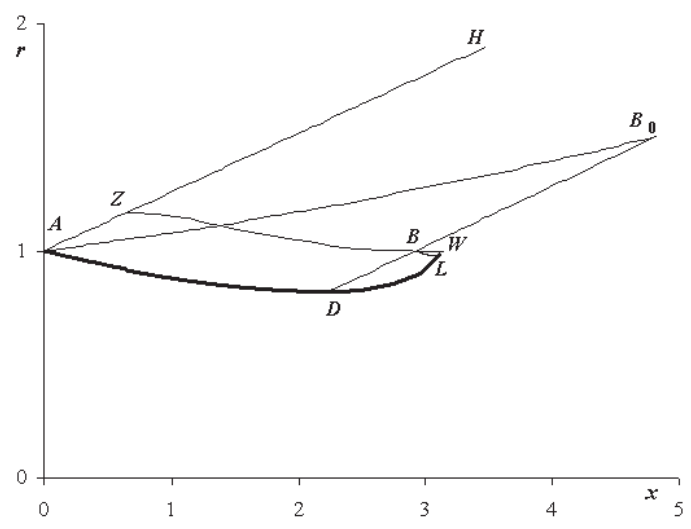

Рис. 5. Результат расчета контура центрального тела для ТуВ с точкой фокусировки выше обечайки. L, A - угловые точки; $B_{0}$ - точка фокусировки; $B$ - точка начала обечайки; AH - равномерная характеристика во внешнем потоке; $L W$ - равномерная характеристика в минимальном сечении; ZB - граница захватываемой струи; $A B_{0}$ - первая характеристика, приходящая в точку фокусировки; $B_{0} B D-$ последняя характеристика, приходящая в точку фокусировки; $D B L$ - область решения задачи Гурса, $A D L-$ контур центрального тела; $B W$ - элемент обечайки

Fig. 5. Results of designing the central body contour for the ducted air inlet with the focusing point over the shell. L, A are the angular points; $B_{0}$ is the focusing point; $B$ is the point of the beginning of the shell; $A H$ is the uniform characteristic in the external flow; $L W$ is the uniform characteristic in the minimal cut set; $Z B$ is the boundary of the captured jet; $A B_{0}$ is the first characteristic moving to the focusing point; $B_{0} B D$ is the last characteristic moving to the focusing point; $D B L$ is the Goursat problem space; $A D L$ is the central body contour; $B W$ is the shell element 
В таблице представлены результаты расчетов. Они показывают, что максимальному расходу ТуВ при прочих равных параметрах соответствует $r_{B_{0}}=1$ - точка фокусировки, лежащая на обечайке. Расход $G$ вычислялся по формуле (3). Исходные данные: $N=100, k=1,4 ; \mathrm{M}_{1}=4 ; \mathrm{M}_{2}=1,2 ; r_{h}=1,9$. Параметр $G_{R E L}$ на полученные результаты не влияет.

Таблица. Влияние положения точки фокусировки $r_{B_{0}}$ на расход $G$ и радиус минимального сечения $r_{L}$ туннельного воздухозаборника

Table. Influence of the focusing point $r_{B_{0}}$ on the flow $G$ and radius of minimal cut set $r_{\llcorner}$of the ducted air inlet

\begin{tabular}{|c|c|c|c|c|c|c|c|c|c|}
\hline$r_{B 0}$ & 1,0 & 1,1 & 1,2 & 1,3 & 1,4 & 1,5 & 1,6 & 1,7 & 1,8 \\
\hline$G$ & 0,106 & 0,091 & 0,076 & 0,062 & 0,047 & 0,033 & 0,021 & 0,011 & 0,003 \\
\hline$r_{L}$ & 0,944 & 0,952 & 0,960 & 0,968 & 0,975 & 0,983 & 0,989 & 0,995 & 0,998 \\
\hline
\end{tabular}

Отметим, что можно провести оптимизацию полученного профиля под выполнение опреде-

\section{СПИСОК ЛИТЕРАТУРЫ}

1. Абрамович Г.Н. Прикладная газовая динамика. - М.: Наука, 1969. - 824 c.

2. Connors J.F., Woollett R.R. Characteristics of flow about axially symmetric isentropic spikes for nose inlet at Mach number 3.85. - NACA: Reports and Memoranda no. E54F08. - 1954. 53 p. URL: http://ntrs.nasa.gov/archive/nasa/casi.ntrs.nasa.gov/ 19930088579.pdf (дата обращения: 20.01.2015).

3. Wallace F.D., Scherrer R. Aerodynamic principles for the design of jet engine induction systems // National advisory committee for aeronautics. - Washington, February 27, 1956. - 185 p. URL: https://archive.org/stream/nasa_techdoc_19650013440/ 19650013440\#page/n0/mode/2up (дата обращения: 20.01.2014).

4. Kennedy E.C. Calculation of axisymmetric isentropic spike surfaces // J. Aerospace Sci. - 1958. - V. 25. - № 7. - P. 463-464.

5. Goldsmith E.L., Smith G.V.F. The performance of some axi-symmetric isentropic centrebody intakes designed for Mach numbers of 2.48 and 3.27. - London: Reports and Memoranda no. 3585. 1966. - 83 p. URL: http://naca.central.cranfield.ac.uk/reports /arc/rm/3585.pdf (дата обращения: 20.01.2015).

6. Аэродинамика и динамика полета магистральных самолетов / под. ред. Г.С. Бюшгенса. - М., Пекин: ИО ЦАГИ, Авиа-издательство КНР, 1995. - 772 с.

7. Артёмов 0.А. Прямоточные воздушно-реактивные двигатели (расчет характеристик). - М.: Компания Спутник+, 2006. $374 \mathrm{c.}$

8. Variational analysis and aerospace engineering // Springer Optimization and Its Applications / eds. G. Buttazzo, A. Frediani. New York, Springer-Verlag, 2009, V. 33. - 518 p.

9. Tactical Missile Aerodynamics: General Topics // Series: Progress in Astronautics and Aeronautics / Ed. M.J. Hemsch. - American Institute of Aeronautics and Astronautics, 1992. V. 141. $-731 \mathrm{p}$

10. Zarchan P. Tactical and strategic missile guidance // Series: Progress in Astronautics and Aeronautics. American Institute of Aeronautics and Astronautics, 2013. - V. 239. - 1026 p.

11. Critical technologies for hypersonic vehicle development. Lecture Series. 2004, 546 p. URL: https://www.cso.nato.int/pubs/rdp. asp?RDP=RT0-EN-AVT-116 (дата обращения: 20.01.2015). ленных конструкционных требований, например, учесть влияние вязкости, как это было сделано при создании профилированных гиперзвуковых сопел для аэродинамической трубы АТ303 [20].

\section{Заключение}

На основе метода характеристик разработана методика построения осесимметричного сверхзвукового туннельного воздухозаборника. Его входное отверстие размещено на боковой поверхности летательного аппарата и не имеет элементов, выступающих во внешний поток. Геометрические характеристики предложенного воздухозаборника единственным образом зависят от исходных данных. Проведенные расчеты показали, что при прочих равных исходных данных положение точки фокусировки на обечайке обеспечивает максимальный расход через предлагаемый воздухозаборник.

12. Ludwig Karl-Heinz. Technik und ingenieure im dritten reich. Dusseldorf: Droste, 1974. - 544 p.

13. Christopher J. The Race for Hitler's X-Planes: Britain's 1945 Mission to Capture Secret Luftwaffe Technology. - UK: The History Press, 2013. - 224 p.

14. Малогабаритный туннельный воздухозаборник воздушно-реактивного двигателя дозвукового летательного аппарата: пат. Рос. Федерация № 134516; заявл. 19.04.13; опубл. 20.11.13. Бюл. № 32. - 2 c.

15. Замарахин В.А., Кирюхин И.В., Швыкин Ю.С. Способ повышения эффективности утопленного воздухозаборника // Оборонная техника. - 2014. - № 10. - С. 15-19.

16. Проектирование туннельных воздухозаборников изоэнтропического сжатия для осесимметричных летательных аппаратов / Е.Я. Брагунцов, Д.А. Внучков, В.М. Галкин, В.И. Звегинцев // Авиация и космонавтика - 2014: Тезисы докладов 13 Междунар. конф. - М.: 17-21 ноября 2014. - СПб.: Мастерская печати, 2014. - С. 111-113.

17. Theory of optimum aerodynamic shapes / ed. A. Miele. - New York, London: Academic press, 1965. - 478 p.

18. Galkin V.M., Volkov Yu.S. Elements of Nozzle Design Optimization / In: Encyclopedia of Mathematics Research / eds. Mathias J.D., Cleaves S.I. - New York: Nova Science Publishers, Inc., 2012. - V. 3. - P. 843-875. URL: https://www.novapublishers. com/catalog/product_info.php?products_id=47969 (дата обращения: 20.01.2015).

19. Волков Ю.С., Галкин В.М. 0 выборе аппроксимаций в прямых задачах построения сопла // Журнал вычислительной математики и математической физики. - 2007. - Т. 47. - № 5. C. $923-936$.

20. Численное проектирование многорежимных осесимметричных гиперзвуковых сопел аэродинамической трубы / С.М. Аульченко, В.М. Галкин, В.И. Звегинцев, А.Н. Шиплюк // Прикладная механика и техническая физика. 2010. - T. 51. - № 2. - C. 90-97.

Поступила 29.01.2015 2. 


\title{
FORMING OF DUCTED AXISYMMETRIC SUPERSONIC AIR INLETS
}

\author{
Vladislav M. Galkin, \\ Tomsk Polytechnic University, \\ 30, Lenin Avenue, Tomsk, 634050, Russia. Email: vlg@tpu.ru \\ Valery I. Zvegintsev, \\ Khristianovich Institute of Theoretical and Applied Mechanics \\ of the Siberian Branch of the Russian Academy of Sciences, 4/1, Institutskaya \\ street, Novosibirsk, 630090, Russia. Email: zvegin@itam.nsc.ru
}

\begin{abstract}
Relevance of the research. The efficiency of fuel chemical energy conversion into mechanical motion of a supersonic aircraft is determined by energy losses due to aerodynamic drag of the aircraft and total pressure losses in the air inlet of jet engine. Therefore, ceteris paribus the aircraft with lower level of these losses will be more energy efficient. Significant increase in energy efficiency is expected from the use of axisymmetric isentropic ducted air inlets. In the framework of the non-viscous flow, a numerical method is offered for these air inlets with a contoured central body and a cylindrical shell designing.

The main aim of the study. Currently there are no designing methods for axisymmetric isentropic ducted air inlets without elements projecting over a cylindrical body of the aircraft. Therefore, the aim of the study is to develop a methodology for designing such inlets. The methods used in the study. Numerical implementation of the characteristics method for isentropic flow is used. The proposed method involves the decision of two problems. In the first problem a flow line around the given focus point of characteristics which starts at a break point of central body is plotted. In the second problem the rest of the contour is calculated using the reversed flow in an annular nozzle with a cylindrical shell. Simultaneously with the decision of the second problem, the position of the shell is determined. The central body can contain angular points at the edges of the contour. If we use an intermediate flow line then the central body will be smooth.

The results. The authors developed the methodology for calculating different axisymmetric supersonic air inlet contours, which geometrical characteristics are uniquely described by the original data.
\end{abstract}

Key words:

Energy efficiency, supersonic flow, ducted axisymmetric air inlet, ideal perfect gas, method of characteristics, isentropic flow.

\section{REFERENCES}

1. Abramovich G. N. Prikladnaya gazovaya dinamika [Applied gas dynamics]. Moscow, Nauka Publ., 1969. 824 p.

2. Connors J.F. Woollett R.R. Characteristics of flow about axially symmetric isentropic spikes for nose inlet at Mach number 3.85 . NACA, Reports and Memoranda no. E54F08, 1954, 53 p. Available at: http://ntrs.nasa.gov/archive/nasa/casi.ntrs.nasa.gov/ 19930088579.pdf (accessed 20 January 2015).

3. Wallace F.D., Scherrer R. Aerodynamic principles for the design of jet engine induction systems. National advisory committee for aeronautics. Washington, February 27, 1956. 185 p. Available at: https://archive.org/stream/nasa techdoc 19650013440/ 19650013440\#page/n0/mode/2up (accessed 20 January 2015).

4. Kennedy E.C. Calculation of axisymmetric isentropic spike surfaces. J. Aerospace Sci., 1958, vol. 25, no. 7, pp. 463-464.

5. Goldsmith E.L., Smith G.V.F. The performance of some axi-symmetric isentropic centrebody intakes designed for Mach numbers of 2.48 and 3.27. London, Reports and Memoranda no. 3585, 1966, 83 p. Available at: http://naca.central.cranfield.ac.uk/reports/ arc/rm/3585.pdf (accessed 20 January 2015).

6. Aerodinamika i dinamika poleta magistralnykh samoletov [Aerodynamics and flight dynamics of aircraft]. Ed. G. S. Byushgens. Moscow, Peking, TSAGI Publ., Avia-izdatelstvo China, 1995. $772 \mathrm{p}$.

7. Artemov 0.A. Pryamotochnye vozdushno-reaktivnye dvigateli (raschet kharakteristik) [Ramjet-rocket engines (calculation of characteristics)]. Moscow, Kompaniya Sputnik+, 2006. 374 p.

8. Variational Analysis and Aerospace Engineering. Springer Optimization and Its Applications. Eds. G. Buttazzo, A. Frediani. New York, Springer-Verlag, 2009, vol. 33, 518 p.

9. Tactical Missile Aerodynamics: General Topics. Series: Progress in Astronautics and Aeronautics. Ed. M.J. Hemsch. American Institute of Aeronautics and Astronautics, 1992, vol. 141, $731 \mathrm{p}$.
10. Zarchan P. Tactical and Strategic Missile Guidance. Series: Progress in Astronautics and Aeronautics. American Institute of Aeronautics and Astronautics, 2013, vol. 239, 1026 p.

11. Critical technologies for hypersonic vehicle development. Lecture Series. 2004, 546 p. Available at: https://www.cso.nato.int/pubs/ rdp.asp? RDP=RTO-EN-AVT-116 (accessed 20 January 2014).

12. Ludwig Karl-Heinz. Technik und Ingenieure im Dritten Reich. Dusseldorf, Droste, 1974. 544 p.

13. Christopher J. The Race for Hitler's X-Planes: Britain's 1945 Mission to Capture Secret Luftwaffe Technology. UK, The History Press, 2013. 224 p.

14. Chevagin A.F., Tretyakov V. F., Kazhan A.V., Kazhan V.G. $M a-$ logabaritnyy tunnelnyy vozdukhozabornik vozdushno-reaktivnogo dvigatelya dozuukovogo letatelnogo apparata [Small-sized tunnel RAM intake subsonic jet aircraft]. Patent RF, no. 134516, 2013.

15. Zamarakhin V.A., Kiryukhin I.V., Shvykin Yu.S. Sposob povysheniya effektivnosti utoplennogo vozdukhozabornika [Way to improve the efficiency of recessed air intake]. Oboronnay a tekhnika, 2014, no. 10, pp. 15-19.

16. Braguntsov E.Ya., Vnuchkov D.A., Galkin V.M., Zvegintsev V.I. Proektirovanie tunnelnykh vozdukhozabornikov izoentropicheskogo szhatiya dlya osesimmetrichnykh letatelnykh apparatov [Design of tunnel axisymmetric diffusion aircraft]. Tezisy dokladov 13 Mezhdunarodnoy konferentsii. Aviatsiya $i$ kosmonavtika2014 [Proc. 13 ${ }^{\text {th }}$ Int. Symp. Aviation and Cosmonautics - 2014]. Moscow, 2014. pp. 111-113.

17. Theory of optimum aerodynamic shapes. Ed. by A. Miele. New York, London, Academic press, $1965.478 \mathrm{p}$.

18. Galkin V.M., Volkov Yu.S. Elements of Nozzle Design Optimization. In: Encyclopedia of Mathematics Research. Eds. Mathias J.D., Cleaves S.I. New York, Nova Science Publishers, Inc., 
2012, vol. 3, pp. 843-875. Available at: https://www.novapublishers. com/catalog/product_info.php?products_id=47969 (accessed 20 January 2015).

19. Volkov Yu.S., Galkin V.M. On the choice of approximations in direct problems of nozzle design. Computational Mathematics and Mathematical Physics, 2007, vol. 47, no. 5, pp. 882-894.

Available at: http://portal.tpu.ru/SHARED/v/VLG/eng/Tab4/ Tab/volkov_galkin_2007.pdf (accessed 20 January 2015).
20. Aulchenko S.M., Zvegintsev V.I., Galkin V.M., Shiplyuk A.N. Numerical design of multimodal axisymmetric hypersonic nozzles for wind tunnels. Journal of Applied Mechanics and Technical Physics, 2010, vol. 51, no. 2, pp. 218-225. Available at: http://portal.tpu.ru/SHARED/v/VLG/eng/Tab4/Tab/ jamtp_2010.pdf (accessed 20 January 2015).

Received: 29 January 2015. 